\title{
Assessment of myocardial strain using strain-encoding (SENC) MRI: comparison of acquisition strategies
}

\author{
El-Sayed H Ibrahim ${ }^{1 *}$, Wolfgang Rehwald², Bradley P Sutton ${ }^{3}$, Sven Zuehlsdorff², Richard D White
}

From 2011 SCMR/Euro CMR Joint Scientific Sessions

Nice, France. 3-6 February 2011

\section{Introduction}

Strain-encoding (SENC) MRI was recently introduced for measuring strain with high-resolution and simple postprocessing (1). Figure-1 shows SENC pulse sequence. In typical SENC sequence, $\mathrm{k}$-space data is acquired line-byline in rectilinear fashion, which results in long scan-time and renders the technique impractical for many applications. Nevertheless, fast imaging techniques, e.g. Radial or Spiral acquisition, allow for reducing scan time while maintaining adequate image quality. In this work, Radial and Spiral acquisitions were implemented in SENC for improved performance. The developed sequences were tested on volunteers and the results were evaluated and compared to standard Cartesian acquisition.

\section{Methods}

Radial and Spiral acquisitions(2) were implemented in SENC, and the different sequences were tested on three volunteers on Siemens Tim-Trio 3.0-Tesla scanner. The imaging parameters were: $\mathrm{FOV}=350 \mathrm{~mm}$, slice-thickness $=$ $10 \mathrm{~mm}$, flip-angle $=15^{\circ}$, \# heart-phases $=25$, and scan time $=17 \mathrm{~s}$. The three sequences were optimized for the

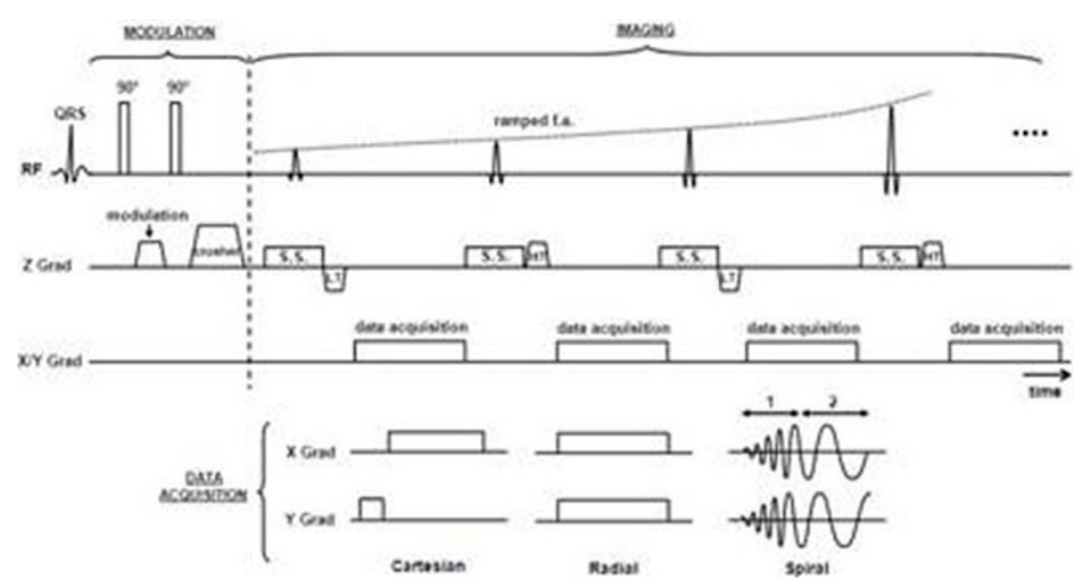

Figure 1 SENC pulse sequence. The pulse sequence consists of two parts: modulation and imaging. The modulation part is composed of two non-selective $90^{\circ} \mathrm{RF}$ pulses, interspersed by a modulation gradient in the slice-selection direction, and followed by a crusher gradient. The imaging part is composed of a series of ramped slice-selective RF pulses, each followed by a demodulation (tuning) gradient in the sliceselection direction, and then data acquisition. Interleaved tunings were implemented to reduce scan time. Data acquisition can be either Cartesian, Radial or Sprial, as shown in the bottom. Spiral gradients were designed to consist of two parts: 1) slew-rate limited and 2) amplitude limited.

'Department of Radiology, University of Florida, Jacksonville, FL, USA

Full list of author information is available at the end of the article

(c) $2011 \mathrm{lbrahim}$ et al; licensee BioMed Central Ltd. This is an open access article distributed under the terms of the Creative Commons Attribution License (http://creativecommons.org/licenses/by/2.0), which permits unrestricted use, distribution, and reproduction in any medium, provided the original work is properly cited. 

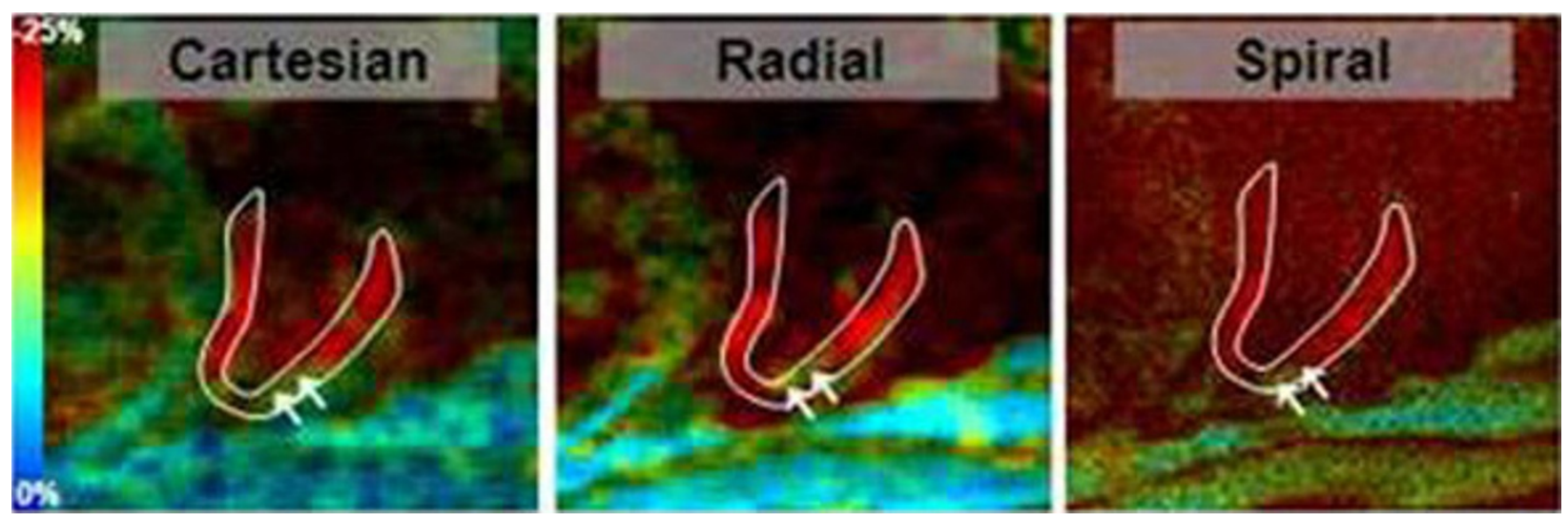

Figure 2 SENC two-chamber images from Cartesian (left), Radial (middle), and Spiral (right) acquisitions showing circumferential strain at endsystole. Carteseian and Radial show similar image quality, while Spiral shows superior resolution. All images show similar contracting pattern in the heart. The arrows point to part of the apical wall showing low strain, which showed in all images.

fixed scan-time. Maximum resolution obtained was 160x160 for Cartesian (80\% phase-encoding coverage) and Radial (128 Radial spokes), and 256x256 for Spiral (10 spiralsx 2 averages). The low-tuning and high-tuning images were combined together as described in(1) to construct the strain images. Strain values were measured at five different points along the lateral left-ventricular wall on all volunteers. Bland-Altman analysis was conducted to compare measurements from different sequences.

\section{Results}

Figure-2 shows example of the acquired SENC images. The strain values measured at the same position were similar in different images, as shown in the strain curves in Figure-3. The Bland-Altman analysis showed no bias between strain measurements from different acquisitions (Figure-4). The mean \pm SD of the (circumferential) strain differences were $0.42 \pm 2.46$ and $-0.25 \pm 1.76 \%$ for the Radial-Cartesian and Spiral-Cartesian differences, respectively. All the differences lied within the $\pm 2 \mathrm{SD}$ limit.

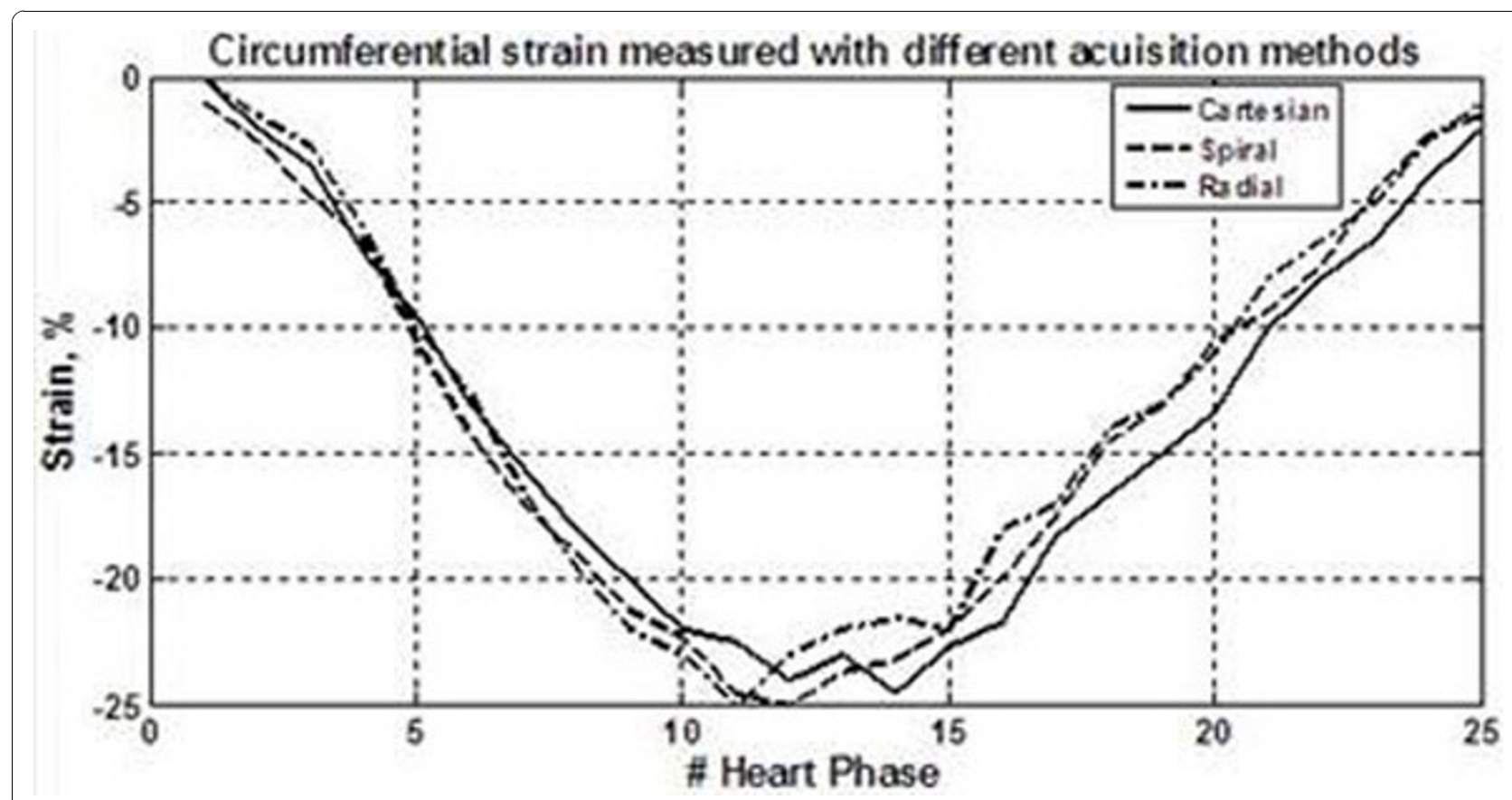

Figure 3 Circumferential strain curves through the cardiac cycle (25 phases) measured from SENC images with Cartesian (solid), dashed (Spiral), and Radial (dot-dashed) acquisitions. The curves show similar contracting pattern irrespective of the acquisition strategy. 


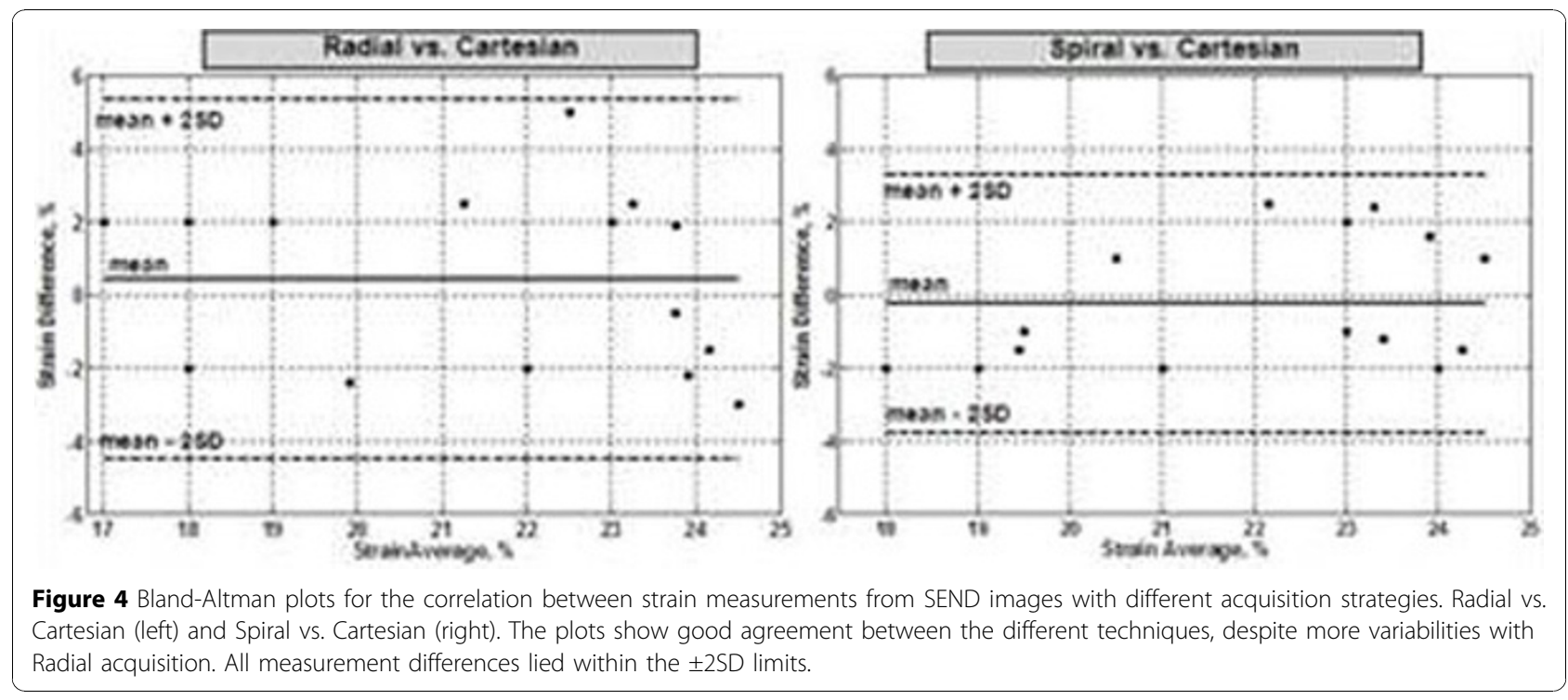

\section{Discussion and conclusions}

Data acquisition strategy (k-space trajectory) affects scan-time and the resulting image-quality in SENC. Image quality was similar in Cartesian and Radial. Less radial spokes can be acquired to reduce scan-time without much affecting image-quality. Due to its acquisition nature, spatial-resolution is compromised in Radial acquisition. For the same scan time, Spiral acquisition allowed for improving resolution by more than $60 \%$ and doubling \# averages, compared to Cartesian or Radial, despite longer reconstruction time. High spatialresolution would allow for accurate measurements in small structures, e.g. thinning myocardial wall, or it can be traded for faster or real-time imaging. The choice of the acquisition-technique depends on patient condition, available scan-time, and imaging features of importance.

\section{Author details}

${ }^{1}$ Department of Radiology, University of Florida, Jacksonville, FL, USA.

${ }^{2}$ Siemens Medical Solutions, MRI Cardiovascular R\&D, Chicago, IL, USA.

${ }^{3}$ Department of Bioengineering, University of Illinois, Urbana-Champaign,

IL, USA.

Published: 2 February 2011

\section{References}

1. Osman, et al: MRM 2001, 324-334.

2. Glover GH: MRM 1999, 412-415.

doi:10.1186/1532-429X-13-S1-P15

Cite this article as: Ibrahim et al:: Assessment of myocardial strain using strain-encoding (SENC) MRI: comparison of acquisition strategies.

Journal of Cardiovascular Magnetic Resonance 2011 13(Suppl 1):P15.

Submit your next manuscript to BioMed Central and take full advantage of:

- Convenient online submission

- Thorough peer review

- No space constraints or color figure charges

- Immediate publication on acceptance

- Inclusion in PubMed, CAS, Scopus and Google Scholar

- Research which is freely available for redistribution

Submit your manuscript at www.biomedcentral.com/submit 